\title{
Qualitative and Quantitative Integrated Modeling for Stochastic Simulation and Optimization
}

\author{
Xuefeng Yan, ${ }^{1}$ Yong Zhou, ${ }^{1}$ Yan Wen, ${ }^{1}$ and Xudong Chai ${ }^{2}$ \\ ${ }^{1}$ College of Computer Science and Technology, Nanjing University of Aeronautics and Astronautics, Nanjing, Jiangsu 210016, China \\ ${ }^{2}$ Beijing Simulation Center, Beijing 100854, China \\ Correspondence should be addressed to Xuefeng Yan; xuefeng.yan@gmail.com
}

Received 21 April 2013; Accepted 17 May 2013

Academic Editor: Neal N. Xiong

Copyright (c) 2013 Xuefeng Yan et al. This is an open access article distributed under the Creative Commons Attribution License, which permits unrestricted use, distribution, and reproduction in any medium, provided the original work is properly cited.

\begin{abstract}
The simulation and optimization of an actual physics system are usually constructed based on the stochastic models, which have both qualitative and quantitative characteristics inherently. Most modeling specifications and frameworks find it difficult to describe the qualitative model directly. In order to deal with the expert knowledge, uncertain reasoning, and other qualitative information, a qualitative and quantitative combined modeling specification was proposed based on a hierarchical model structure framework. The new modeling approach is based on a hierarchical model structure which includes the meta-meta model, the meta-model and the high-level model. A description logic system is defined for formal definition and verification of the new modeling specification. A stochastic defense simulation was developed to illustrate how to model the system and optimize the result. The result shows that the proposed method can describe the complex system more comprehensively, and the survival probability of the target is higher by introducing qualitative models into quantitative simulation.
\end{abstract}

\section{Introduction}

Stochastic simulation has become a highly effective and essential part of all scientific fields to analyze, reconstruct, and optimize the objective world without the need to perform experiments on a physical product or an actual system. In theoretical and experimental research, it has become another important way to reveal the internal and essential laws of the real world. To study and gain insight into real phenomena, a stochastic model should be constructed for some particular purpose at an appropriate level of abstraction or fidelity.

In the field of stochastic simulation, whenever we mention "qualitative model," the phrase "quantitative model" will naturally come to mind. In fact, "simulation model" generally refers to a quantitative model if not particularly described, and most research is based on the mathematical model [1]. Precise mathematical models are built to describe the system structure and behavior, especially the logic and functionality on the timeline. The simulation is carried out by solving the equations in a numerically calculated fashion. The simulation results rely on the accuracy of the models. However, the mathematical perfection is not representative of the authenticity of the system and the subtle experiential meaning of the real world cannot be modeled by mathematical equations. On the other hand, the objects we studied, such as aircraft, weapons, and space systems, are increasingly complex. This is particularly true of giant, complex system. We can only have or create some of the mathematical models with certain accuracy. It is almost impossible to construct all the quantitative models and complete their Verification, Validation and Accreditation (VVA). Furthermore, not all of the simulation requires a precise mathematical model. For example, sometimes we are only interested in the macroevolution trend of a system, rather than time-specific values.

The symbol qualitative model can contain various forms of information and has reasoning and learning ability. The structure and behavior of the actual system are described in an abstract form, focusing on the causality and not on mathematical equations. It is widely used in many fields associated with physics, chemistry, ecology, biology, fault diagnosis, mechanical manufacturing, industrial systems, and Artificial Intelligence (AI) [2]. We can see that the combination of qualitative and quantitative attributes shows promise for stochastic simulation. Many scholars have made important 
progresses in this field [3-9]. Due to the direct usage of existing expertise, qualitative and quantitative integrated methods have many significant advantages.

(1) When it is difficult to build all the quantitative models and the stochastic simulation cannot be constructed because some models are lacking, the qualitative model could be a necessary complement.

(2) Qualitative modeling is effective for some fields where most of the knowledge is expressed by symbols, language, or graphics directly.

(3) When we are just interested in the macroevolution or the essential qualitative phenomenon, it is not necessary to occupy a large number of computing time and resources for quantitative simulation.

(4) The static structure of the simulation can be organized based on qualitative models and at run-time, qualitative models can intelligently choose the better execution branch or data based on the schedule engine. Different Detail of Level (DOL) resolution can be constructed for a system at different abstraction levels.

(5) The traditional evaluation and optimization can be innovated because the qualitative mode is a part of the simulation and online assessment could be made.

We can see that the qualitative model brings an unprecedented opportunity to improve traditional stochastic simulation. But it also faces with the following challenges.

(1) There are a large number of different types of qualitative models in different application fields, and the requirements, interfaces, and forms are varied.

(2) The qualitative modeling methods and symbolic languages are also diverse in different applications fields. These heterogeneous models are incompatible with each other and it is difficult to simulate together.

(3) The loose and redundancy qualitative models should be integrated with the rigorous quantitative models to form the stochastic simulation with a precise logical structure. Many effects are needed in qualitative and quantitative hybrid simulation engines [10].

There are lots of classic researches in quantitative modeling, such as the specification named Discrete Event Systems Specification (DEVS) for discrete event systems and COllaborative SIMulation (COSIM) for multidisciplinary virtual prototype modeling and simulation [11-16]. In order to deal with expert knowledge, uncertain reasoning, and other qualitative information, a qualitative and quantitative integrated modeling specification and the theoretical framework for stochastic simulation and optimization are significant. In this paper, a hierarchical model structure is proposed, including meta-meta model, meta-model, and the high-level model. The qualitative and quantitative heterogeneous model and integrated relationship were described at a higher abstraction level. The description logic system is defined for the framework based on the formal description and verification of the modeling specification.
The rest of this paper is organized as follows. Section 2 briefly introduces the related researches of qualitative and quantitative modeling for stochastic simulation. In Section 3, a qualitative and quantitative integrated modeling specification is presented, including the modeling framework, description logic system, and formal definitions. Section 4 proves the self-close feature of the models. In Section 5, a qualitative and quantitative mixed stochastic defense system is modeled and simulated. Section 6 draws related conclusions and points out future work.

\section{Related Works}

2.1. Qualitative Model in Stochastic Simulation. A complex stochastic simulation is always composed of various subsystems. To analyze and optimize the performance, qualitative models have been investigated and applied to more and more fields [17]. In [1], tactical decision making based on fuzzy logic was applied to an underwater vehicle in an engagement-level simulation. A light torpedo and a submarine were modeled based on DEVS and the submarine model calls the fuzzy logic model to conduct a tactical decision. The fuzzy logic was implemented as the Python script tactic description file. By adopting the fuzzy logic, a smoother result was obtained than fixed established tactics and the survival possibility of the submarine was enhanced. SHAO Chen-xi believed that qualitative modeling and simulation makes it feasible to deal with incomplete information. He summarized classic technologies such as fuzzy qualitative simulation, reduction reasoning, noncausal reasoning, causal-based reasoning, diagram-based reasoning, structural data-based modeling, and qualitative space-based reasoning. The application fields were also introduced, including ecology, mechanical manufacturing, medical research, and hybrid nonlinear systems [18]. In [19], a modeling method based on the relationship and transmission of effect between nodes was introduced. Based on the strength of the definition of cause and effect, a flexible modeling method was designed for graph-based qualitative systems. Nonautonomous systems changing with time can be analyzed using the new method. A causal relationship chart model of the quality risk based on integrating casual is proposed in [20]. An example is used to demonstrate the entire risk evolution triggered by changes in one quality factor, simulating the evolution process in accordance with reality. The application indicates that the proposed method can serve as a useful experimental tool for decision making in facing risks by highway construction project teams. A qualitative simulation model of changing processes of customer churn is constructed based on the causality graph in [21]. The qualitative simulation and random behavior extraction can be executed repeatedly to predict the changing process of customer churn. After analyzing three qualitative simulation methods, noncausality reasoning, causality reasoning, and cellular automata, $\mathrm{Hu}$ and Xiao discussed the complexity characteristics of a management system and introduced their qualitative simulation [22]. 
2.2. Qualitative and Quantitative Integrated Modeling in Stochastic Simulation. Many important theories and applications show that qualitative and quantitative combined methodologies have extremely important significance and promote value for stochastic simulation. A considerable amount of researches have been performed in recent years and many meaningful outcomes have been put forward in different domains. In [23], the proposal and recent development of the "meta-synthetic methodology from qualitative to quantitative" were introduced in detail. Subsequently, many researchers concentrated on qualitative and quantitative combined modeling for stochastic simulation.

FAN Shuai proposed a qualitative and quantitative synthetic modeling method by extending the System High Level Modeling Language for multidiscipline virtual prototype. The qualitative knowledge is modeled based on a cause and effect diagram [3]. Then, the qualitative and quantitative integration simulation architecture was designed, including mixed schedule strategies, time management, and date interaction methods [4]. Qualitative models can be built using the Fuzzy Inductive Reasoning paradigm in Modelica. The qualitative models make use of fuzzy inductive reasoning. The qualitative and quantitative models can be combined to simulate concurrently. A textbook example of a hydraulic position control system and the human cardiovascular system were adopted to demonstrate the approach. The hemodynamics was modeled by quantitative models and the central nervous system was described using qualitative FIR models [5]. In [6], a qualitative and quantitative hybrid model was established for business factors evaluation. Statistical values based on propagation and combination of effects of business factors were introduced in the simulation. Li et al. proposed architecture of qualitative and quantitative comprehensive modeling and studied joint simulation technology for complex systems. In [7], a visualized fuzzy qualitative knowledge modeling method fuzzy causal directed graph was designed, which included the grammar, reasoning, and conversion of qualitative and quantitative models. In [8], a new technique, Q2, was proposed to combine qualitative and quantitative models and was demonstrated in the case of a Finnish transport sector that faces severe pressure to cut $\mathrm{CO}_{2}$ emissions. Liu et al. studied the integration of qualitative reasoning and quantitative simulation including the acquisition, management, and expression of qualitative and quantitative knowledge. Then, an integrated diagnosis inference method was proposed and validated with the test-fire data of complicated systems [9].

Some of the previous studies can be applied to continuous systems, discrete systems, or continuous discrete hybrid system modeling, respectively. They focus on the combination of qualitative and quantitative models from specific application fields. Some researchers achieve qualitative and quantitative combined modeling and simulation based on commercial software tools.

2.3. The COllaborative SIMulation Modeling Theory. COSIM is actually an application of Model Driven Architecture (MDA) for stochastic modeling and simulation. It is mainly a framework for simulation of complex systems, especially complex product virtual prototypes based on heterogeneous models of different fields. In [16], the modeling specification was proposed as the infrastructure of COSIM which is referred to as the Meta Modeling Framework $\left(M^{2} F\right)$. Here, the meta-meta-model, meta-model, and model of different levels were defined to describe the systems. The modeling specification is independent of the realization, which means were that various modeling methods could be involved in the simulation and can be unified with the $\mathrm{M}^{2} \mathrm{~F}$ without considering implementation issues. Meanwhile, $\mathrm{M}^{2} \mathrm{~F}$ serves as a shield to the differences of the modeling methods and forms with higher abstraction than the heterogeneous model.

2.4. Summary. We can see from the aforementioned that there are many researches on optimization of complex stochastic simulation based on qualitative models. The latest research involves the study of a specific application in a given a field based on a selected theory. Many theories such as reduction reasoning, noncausal reasoning, and causal-based reasoning are considered, respectively. Some researchers achieve qualitative and quantitative combined modeling and simulation based on a commercial software tools. There are mainly two ways to integrate the qualitative and quantitative models, microintegration and macrointegration. The former one extended quantitative description method for qualitative knowledge, usually in the form of qualitative and quantitative mixed algebra equation, such as interval values expression and fuzzy mathematical. Although some qualitative knowledge is used, they are not the systematic qualitative modeling approaches. The later one is the integration of qualitative models and quantitative models of the different parts of the system. For example, qualitative model and quantitative model can be organized together to form the whole simulation system. These methods are mainly integrating different models in particular application, and few of them consider the problem from the aspects of modeling specification. So, a further solution is needed based on the existing theories and techniques.

\section{Qualitative and Quantitative Integrated Modeling Specification}

3.1. General View of the Qualitative and Quantitative Integrated Model. Before further details, let us first briefly illustrate the general view of the qualitative and quantitative integrated model to be built. We describe a system from the perspectives of static structure and dynamic behavior, based on three types of Interface which is the solid basis of our modeling methodology. The static structure refers to the internal factors, their structure, and interrelationship, for example, the input and output interfaces and their connection relation and the organization structure of the subsystem, and so forth, as shown in Figure 1. The data exchange between the models is archived through the PortItems, and the collection of PortItems with same type is called Port. The set of Ports is called Interface. The three Interfaces (qualitative, quantitative, and event interface, resp.) will have complex internal and external relations with each other, and this is one 


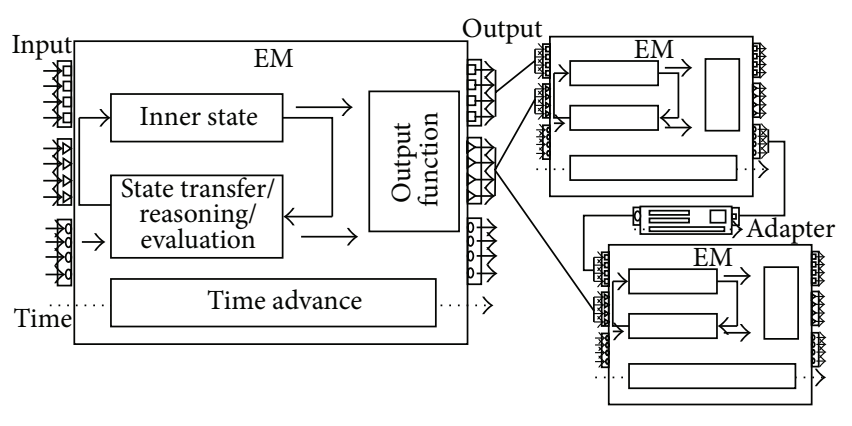

$\square$ Quantitative PortItem $\quad$ Qualitative PortItem

$\triangleright$ Event PortItem

FIGURE 1: Modeling the complex system with component-oriented qualitative and quantitative integrated model.

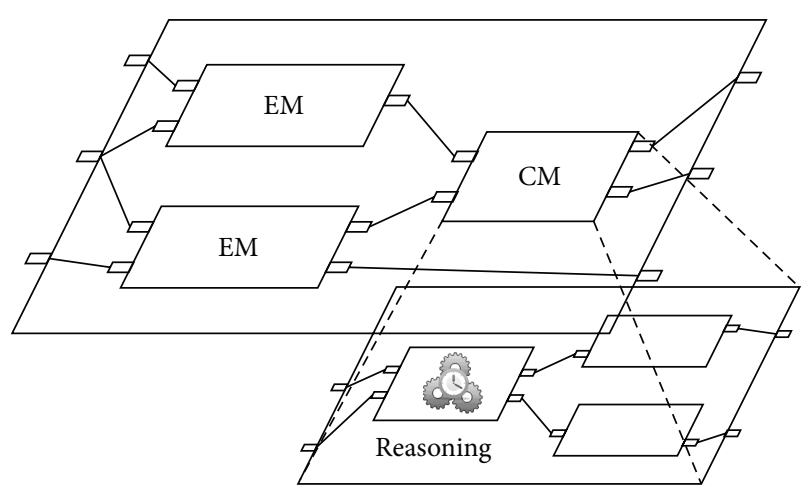

Figure 2: A simulation system integrated by qualitative and quantitative models.

of the focus points in this paper. To describe the temporal logic and simulation process with the time advancing, the state and its transfer, interaction situation, and event flow will be modeled as dynamic behavior, and the reasoning or evaluation functionality will also be involved if needed. We can observe a corresponding output segment from the output interface when some data is set from the input interface, taking the data context into account.

A complex system as a whole is composed by many interconnected and interacted parts, and it can be further divided into smaller and simpler subsystems. It is modeled by component-oriented models with a hierarchy structure. Two types of component model with different structure and size are defined to describe the system, named the element model $(E M)$ and composition model $(C M) . E M$ is the smallest one which cannot be divided any more, while the $C M$ is assembled by EMs and/or smaller CMs according to specific simulation logic by connecting their Interfaces, and then they can collaborate with each other based on an accurate information flow with specific semantics, as shown in Figure 2. In fact the entire simulation system itself is the biggest $\mathrm{CM}$, with a special reasoning component to optimize the simulation process and policy decision based on execution data, history data, and expertise.

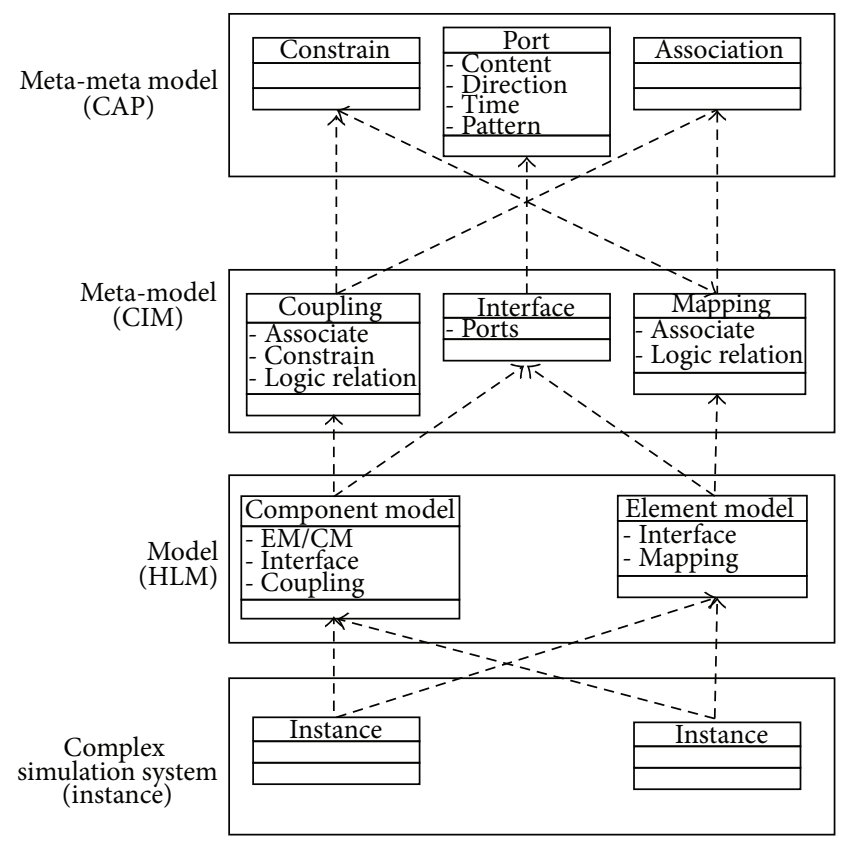

Figure 3: The hierarchical model structure of $\mathrm{Q}^{2} \mathrm{M}^{2} \mathrm{~F}$.

3.2. Qualitative and Quantitative Integrated Meta Modeling Framework. Based on $\mathrm{M}^{2} \mathrm{~F}$, a Qualitative and Quantitative Integrated Meta Modeling Framework $\left(\mathrm{Q}^{2} \mathrm{M}^{2} \mathrm{~F}\right)$, consistent with (MDA) and the rationale of a layered model structure in Meta Object Function (MOF), is defined as a four-layer model framework, as shown in Figure 3. The descriptions of the layers are as follows.

(1) Meta-Meta Model Layer. The prototypes and rules of the meta-model are defined with the highest abstraction level, including Port, Association, Constrain (CAP). The basic factor and its semantics to describe the data structure and knowledge are also defined, just as the basic data type is defined in a programming language.

(2) Meta-Model Layer. The instance of the meta-meta model, Mapping, Interface, Coupling (CIM), defines the basic factor to define a qualitative and quantitative mixed model. It is similar to defining a data structure or class.

(3) Model Layer. The instance of the meta-model, is used to describe the models (so called High-Level Model, HLM) of a specific application field. For example, class "Pilot," a model of the reasoning portion of an expert system, and so forth.

(4) Instance. The instance of the model defines the value of specific parameter or the reasoning part with specific rules, for example, "Pilot Obama."

In $\mathrm{Q}^{2} \mathrm{M}^{2} \mathrm{~F}$, the basic factors in the meta-meta layer are the same as COSIM, but the connotations are redefined to support qualitative and quantitative combined modeling. Logical Relation $(L R)$ is added to the meta-model layer 
to describe the relation of qualitative knowledge. The interaction between qualitative knowledge and quantitative data is added in Mapping and Coupling. Accordingly, in the model layer instance these factors are also defined.

3.3. The Description Logic System for $Q^{2} M^{2} F$. Description logic is used to represent the domain knowledge using a group of structural operators. Knowledge is expressed by concepts and relationships based on the formal reasoning which can be achieved [24-26]. In order to describe the basic factors and their relationships in $Q^{2} M^{2} F$, a description logic system, $A L C_{C}$, is defined based on the classical description logic language, Attributive concept Language with Complements (ALC). The syntactic and semantic facets of $\mathrm{ALC}_{C}$ are defined as follows:

\section{$C, D::=C|T| \perp|\neg C| C \sqcap D|C \sqcup D| \forall R . C \mid \exists R . C$,}

where

$C, D$ : the elementary concept. In $Q^{2} M^{2} F$, the term "element" refers to the smallest atomic model,

$R$ : the elementary binary relation,

$\mathrm{T}$ : the universal concept,

$\perp$ : the bottom concept,

$\neg C$ : the negative concept of $C$,

$C \sqcap D$ : the intersection of $\mathrm{C}$ and $\mathrm{D}$,

$C \sqcup D$ : the union of $\mathrm{C}$ and $\mathrm{D}$,

$\forall R$.C: restricted universal quantification,

$\exists R$.C: restricted existential quantification.

The knowledge base of $A L C_{C}$ is composed of $\left\langle T_{C}, A_{C}\right\rangle$. $T_{C}$ is a finite set of inclusion assertion $\left(T_{b o x}\right)$, and it is also known as a set of terminology axioms. $A_{C}$ is a finite set of instance assertion $\left(A_{b o x}\right)$. It is composed of elementary conception $($ ElemC) and elementary relationship (ElemR), as follows:

$A_{C}=\langle$ ElemC, ElemR $\rangle$.

ElemC $=\{$ Data, Knowledge, Event, Input, Output, Time, Real, Pattern, Association, streig (streig, which means "tied or bound" in ancient Latin. Here it is used to represent a constraint.), $T_{0}$, DataType, KnowledgeType, EventType, STATE, statTF, ID, $\left.M_{x}\right\}$

ElemR $=\{$ has_a, part_of, domain_of, range_of, isa_function, isa_relation_on, content_of, direction_of, time_of, element_of $\}$,

where

Data, Knowledge and Event: quantitative data, qualitative knowledge and event, respectively;

Input and Output: the direction of information flow;

Time: the effective time of the information flow;

Real is the real numbers;

Pattern: the overall scheme of information;
Association and streig: the Association relationship and constraint, respectively;

$T_{0}$ : the initial time;

STATE and statTF: the state and its transfer, respectively;

ID: the index set of the subcomponents;

$M_{X}$ : the set of subcomponents;

has_a and part_of: two inverse elementary relationships, expressing the belonging relationship between the elements of the sets;

domain_of and range_of: the domain and range of the relation;

isa_function: a common function;

isa_relation_on: a binary relation;

content_of: the information of a meta-meta model;

direction_of: the direction of the information;

element_of: the relationship between EM and CM.

More complex conceptions and relationships can be derived from the basic definition mentioned earlier, and the factors at each level in $Q^{2} M^{2} F$ can be described and verified formally.

3.4. Meta-Meta Model (CAP). Qualitative and quantitative meta-meta model is the top level of abstraction of the system model. Port is a meta-port composed by Content, Direction, Time, and Pattern and is used to describe the information interaction with other simulation models or the external environment. Content is all the information interacting between the simulation models through the Port which will affect the simulation process or result. Content can be quantitative data, event, or qualitative knowledge. Direction indicates the transfer direction of the information. Time refers to the position and effective range on the timeline. The value range $T$ is a subset of the positive real numbers $\mathrm{R}^{+}$. Pattern describes the overall pattern of information contained by meta-ports throughout the simulation timeline. It is an enumerable sequence of a set of numerable/innumerable $\langle$ content, time $\rangle$ couples. The formal definition of Port is as follows:

$$
\begin{aligned}
& \text { Port } \equiv \exists \text { has_a.Content } \square \quad \exists \text { has_a.Direction } \square \\
& \exists \text { has_a.Time } \square \exists \text { has_a.Pattern } \\
& \text { Content } \equiv \text { Data } \sqcup \text { Knowledge } \sqcup \text { Event } \\
& \text { Direction } \equiv I n p u t \sqcup \text { Output } \\
& \text { Time } \sqsubseteq \text { Real } \\
& \text { Pattern } \sqsubseteq \text { Content } \times \text { Time. }
\end{aligned}
$$

(Meta) Association is used to describe the numeri$\mathrm{cal} /$ symbolic relationship of information contents between meta-ports. The association represents the direction of the Content, and most of the association is a one-to-one mapping. In quantitative models, the association is reflected as a mapping relationship between quantitative data on the metaports. In qualitative models, it is the connecting relationship 
between qualitative knowledge. Multiple associated ports may also exist, which represent the convergence or distribution of the information flow. The formal definition is

$$
\begin{aligned}
& \text { Association } \sqsubseteq \text { Port_Content } \times \text { Port_Content } \\
& \text { Port_Content } \equiv \text { Content } \sqcap \exists \text { part_of.Port. }
\end{aligned}
$$

Constrain describes the properties of specific Port, including differences in Direction, Time, and Pattern, especially for the ports where Association exists. There are two Constraints, Quantitative Constraint and Qualitative Constraint. By setting constraints on the Direction, Time, and Pattern, the solution logic, temporal order, and modeling mechanism of heterogeneous models can be unified in one simulation system. Constraint will be implemented according to the interior physical mechanism or the state transfer function in the lower layer HLM. The conception of Constrain is defined as

$$
\begin{aligned}
& \text { Port_Direction } \equiv \text { Direction } \sqcap \forall \text { part_of.Port } \\
& \text { Port_Pattern } \equiv \text { Pattern } \sqcap \forall \text { part_of.Port } \\
& \text { Port_Time } \equiv \text { Time } \sqcap \forall \text { part_of.Port } \\
& \text { Constrain } \equiv \text { streig } \sqcap\left(\exists i s a_{-}\right. \text {function.Port_Direction } \\
& \sqcup \exists \text { isa_function.Port_Pattern } \\
& \sqcup \exists i s a_{-} \text {function.Port_Time). }
\end{aligned}
$$

In summary, the concept of CAP is defined formally as

$$
\begin{aligned}
& \text { CAP } \equiv \quad \exists \text { has_a.Port } \sqcap \quad \exists \text { has_a.Association } \sqcap \\
& \exists \text { has_a.Constrain. }
\end{aligned}
$$

3.5. Meta-Model (CIM). We define PortItem, Ports, and Interface as instances of Port in the CIM model. PortItem is consistent with Port, Ports are defined as a collection of PortItems of the same type, and the Interface is defined as a group of Ports with similar properties. This is formally defined as

PortItems $\equiv \exists$ part_of.CAP $\sqcap$ Port
PortItem $\equiv \exists$ part_of.PortItems
Interface $\equiv$ PortItems $\sqcap((\forall$ part_of.PortItems $(x) \rightarrow$
$\exists$ part_of.x $\sqcap$ Direction $\rightarrow \quad$ Input $) \quad \forall$ part
of.PortItems $(x) \rightarrow \quad \exists$ part_of.x $\sqcap$ Direction $=$
Output $)) \sqcap \quad(\forall$ part_of.PortItems $(x) \sqcap \quad \forall$ part
of.PortItems $(y) \rightarrow \exists$ part_of.x $\sqcap$ Pattern $=$
$\exists$ part_of.y $\sqcap$ Pattern $).$

Association and Constrain are essentially interdependent of each other. The former characterizes the existence of the information relationship between the Ports, while the latter adds a limitation on the relationship. There are three instances, Mapping, Logical Relation, and Coupling, in the meta-model inherited from both Association and Constrain.

Mapping, a coinstance of Association and Constrain, is a relationship between the input and output sets of an element model (EM). Figure 4 shows three typical Mappings, the state transfer functions between quantitative PortItems (map), logical relationship between qualitative PortItems (connect), and transform between quantitative and qualitative PortItems. The definition is as follows:

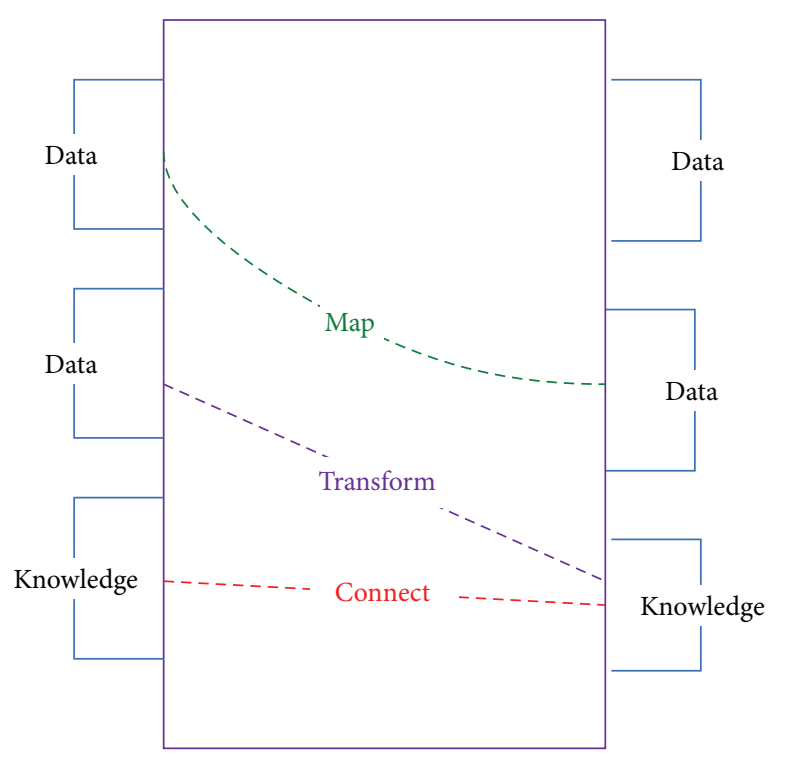

Figure 4: Three typical Mappings in an EM.

Mapping $\equiv$ Maps $\sqcup$ Connects $\sqcup$ Transforms

Maps $\equiv \exists$ part_of CAP $\sqcap$ Association $\sqcap \exists$ domain of.( $\exists$ content_of.Data $\sqcap \exists$ part_of( $\exists$ direction_of.Input $))$ $\sqcap \exists$ range_of.( $\exists$ content_of.Data $\Pi \exists$ part_of( $\exists$ direction of.Output))

Connects $\equiv \exists$ part_of.CAP $\sqcap$ Association $\sqcap \exists i s a_{-}$ relation_on.( $\exists$ content_of.Knowledge)

Transforms $\equiv \exists$ part_of.CAP $\sqcap$ Association $\Pi$ $((\exists$ domain_of.( $\exists$ content_of.Data $) \quad \sqcap \quad \exists$ range_of. $(\exists$ content_of.Knowledge $)) \sqcup((\exists$ domain_of.( $\exists$ content_of. Knowledge $) \sqcap \exists$ range_of.( $\exists$ content_of.Data $)))$.

The relationship between the qualitative Ports is not necessarily expressed via functions; general logical relationships may exist. Logical Relations mainly depicts the qualitative relationship between Ports and the static logical structure of an EM. Consider

LogRelation $\equiv\langle$ connect $|$ connect $\in\left\{\right.$ Interface $_{i}$. Content $\} \times\left\{\right.$ Interface $_{j}$. Content $\}, i \neq j$, Interface I $_{j}$, Interface $\left._{i} \in E M \cup C M\right\rangle$.

Coupling is the interaction between the Ports containing the Associations and Constraints. In addition, it should be noted that the ports associated by Coupling are not just the ports of the submodels within a composite model. Associations could also exist between the output ports of the submodels and the output ports of its superior composite model. Similarly, the input ports of a composite model can be associated with the input of its submodel. The formal definition of Coupling is

Coupling $\equiv$ Coupling_maps $\sqcup$ Coupling_connects

Coupling_maps $\equiv \exists$ part_of.CAP $\sqcap$ (Association $\sqcap$ Constraint) $\sqcap \exists$ domain_of.( $\exists$ content_of.Data $\sqcap \exists$ part of.( $\exists$ direction_of.Input) $) \sqcap \quad \exists$ range_of.( $\exists$ content_of. Data $\sqcap$ ヨart_of.( $\exists$ direction_of.Output)) 
Coupling_connects $\equiv \exists$ part_of.CAP $\sqcap$ (Association $\square$ Constraint) $\square \quad \exists$ isa_relation_on.(ᄏcontent_of. Knowledge).

In summary, the concept of CIM is defined formally as

$C I M \equiv \exists$ has_a.Interface $\Pi \exists$ has_a.Mapping $\sqcap$ Ghas a.Coupling.

3.6. The Hierarchy Model of a Simulation System (HLM). A variety of heterogeneous simulation functionalities are described as standard models using an interface-based modeling strategy. Simulation is achieved via the combination and collaboration of components. In the model layer, the simulation model, named high level model (HLM), will be instanced from three basic factors defined at the meta-model layer. There are two types of qualitative and quantitative mixed simulation models, the Element Model (EM) and the Composite Model $(C M)$.

As the smallest model which cannot be divided any more, EM, consists of Interface, Mapping, and Connecting, the definition is:

EM : $\langle\{$ Interface $\},\{$ Mappings, Connectings $\}\rangle$.

More specifically,

$E M \equiv \exists$ has_a. Init) $\sqcap \exists h a s_{-} a .\left(i P_{d}\right) \sqcap \exists h a s_{-} a .\left(i P_{k}\right) \sqcap$ $\exists$ has_a. $\left(i P_{e}\right) \sqcap \exists$ has_a. $\left(o P_{d}\right) \sqcap \exists h a s_{-} a .\left(o P_{k}\right) \sqcap \exists h a s_{-}$ a. $\left(o P_{e}\right) \sqcap \exists h a s_{-} a .(S T A T E) \sqcap \exists h a s_{-} a_{\text {. }}($ stat TF $) \sqcap \exists h a s_{-}$ a. $(T)$,

where

Init $\equiv \exists$ part_of.CIM $\sqcap$ In_PortItem $\sqcap \exists$ has_a. $(\exists$ time_of. $\left.T_{0}\right) \sqcap \exists$ has_a.DataType

$i P_{d} \equiv \exists$ part_of.CIM $\sqcap$ In_PortItem $\sqcap \exists$ has_a. $(\exists$ content_of.Data) $\sqcap \exists$ has_a.DataType

$i P_{k} \equiv \exists$ part_of.CIM $\sqcap$ In_PortItem $\sqcap \exists$ has_a. $(\exists$ content_of.Knowledge) $\sqcap \exists$ has_a.KnowledgeType

$i P_{e} \equiv \exists$ part_of.CIM $\sqcap$ In_PortItem $\sqcap \exists$ has_a. $(\exists$ content_of.Event) $\sqcap$ ヨhas_a.EventType

$o P_{d} \equiv \exists$ part_of.CIM $\sqcap$ Out_PortItem $\sqcap \exists$ has_a. $(\exists$ content_of.Data) $\sqcap \exists$ has_a.DataType

$o P_{k} \equiv \exists$ part_of.CIM $\sqcap$ Out_PortItem $\sqcap \exists$ has_a. $(\exists$ content_of.Knowledge) $\square \exists$ has_a.KnowledgeType

$o P_{e} \equiv \exists$ part_of.CIM $\sqcap$ Out_PortItem $\sqcap \exists$ has_a. $(\exists$ content_of.Event) $\sqcap \exists$ has_a.EventType.

STATE represents a specific mapping between the input and output Ports. At any time $t$ on the timeline $T$, the simulation model has only one state, csModelState $(t)$, and the formal definition is

STATE $\equiv \exists c s$ ModelState. $T$,

where

StatTF: state transfer refers to the migration process stimulated by external action or internal factors;

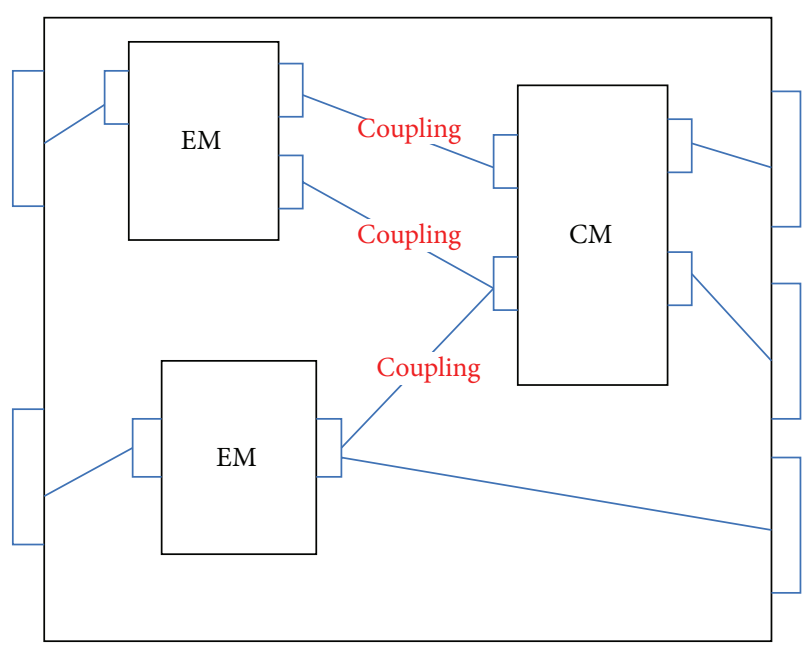

FIGURE 5: The structure of a CM.

StatTF $=\langle$ STATE $\times i P e \rightarrow S T A T E \times T \times o P e| T \subseteq$ $\left.R^{+}\right\rangle$.

A Composite Model (CM) is composed of several EMs and/or CMs with smaller granularity as shown in Figure 5. The formal definition is as follows:

$$
\begin{aligned}
& C M \equiv \exists \text { has_a.(Para) } \sqcap \exists h a s_{-} a . \text { (Init) } \sqcap \exists h a s_{-} a .\left(i P_{d}\right) \sqcap \\
& \exists \text { has_a. }\left(i P_{k}\right) \sqcap \exists h a s_{-} a .\left(i P_{e}\right) \sqcap \exists h a s_{-} a .\left(o P_{d}\right) \sqcap \exists h a s_{-} \\
& \text {a. }\left(o P_{k}\right) \sqcap \exists h a s_{-} a .\left(o P_{e}\right) \sqcap \exists h a s_{2} a .(T) \sqcap \exists h a s_{\text {_a }} \text {. }(I D) \\
& \sqcap \exists h a s_{-} a .\left(M_{x}\right) \sqcap \exists h a s_{-} a .(C P L s) \quad \sqcap \exists h a s_{-} a .(S I T U A) \\
& \Pi \exists \text { has_a. (EvntFL). }
\end{aligned}
$$

Similarly with $E M, C M$ also has a parametric interface, initialization interface, data input and output interfaces, event input and output interfaces, knowledge input and output interfaces, the state and its transfer, and the time-base. They are defined as earlier. $C M$ has three other factors, $I D, M_{X}$, SITUA, EvntFL, and $C P L_{S}$, which do not appear in $E M$, as follows:

$I D$ : the index set of the sub-EM/sub-CM in a $C M$,

$M_{X}$ : the set of sub-EMs and sub-CMs in a $C M$,

CPLs: the Coupling sets in a CM,

SITUA: the sets of interaction situation,

EvntFL: event flow in a CM.

We can see that $C M$ is a self-nested composite model. Besides the element model, EM, which can no longer be divided, it can also include other composition models. In fact, the whole simulation system itself is the largest $C M$.

\section{The Self-Closed Feature of Qualitative and Quantitative Integrated Model}

We can find that the essential difference between the EM and $C M$ is whether or not an internal structure exists. $E M$ describes the internal content of a model via mappings, while $C M$ describes its interior structure and interactions among 
subcomponents. Formally, there are few differences between the two models, but we can note that the formalism of a $C M$ actually has a self-closed structure. Although the internal structure of a $C M$ might be very complicated, a $C M$ should be reused just like an $E M$ in a more complex $C M$. Therefore, in order to ensure reusability, we need to affirm the self-closed feature between the EM and CM. That is, a complicated CM combined by sub-EM and/or sub-CM has the same schema as its subcomponents. On the contrary, the subcomponents decomposed from a $C M$ has the same schema with the original CM.

Definition 1 (Component Communication Graph (CCG)). Assume $C$ is a simulation component (CM or EM). Let directed graph $G_{C}=\left\langle V_{C}, E_{C}\right\rangle$ be the $C C G$ of $C$. Consider

$V_{C}=$ input_interface $(C) \cup$ output_interface $(C)$,

input_interface $(C)=\{x \mid(E M(C) \wedge \operatorname{input}(x, e)) \vee$ $\exists e(E M(e) \wedge$ element_of $(e, C)) \wedge \operatorname{input}(x, e))\}$,

input_interface $(C)$ is the set of all input interfaces of $C$. If $C$ itself is a $C M$, all input interfaces of the internal subcomponents are the same as well,

output_interface $(C)=\{x \mid(E M(C) \wedge$ output $(x, e)) \vee$ $\exists e(E M(e) \wedge$ element_of $(e, C) \wedge$ output $(x, e))\}$.

The edge set $E_{C}$ is

$E_{C}=\left\{\langle x, y\rangle \mid\left(E M(C) \wedge\langle x, y\rangle \in\right.\right.$ Mapping $\left._{C}\right) \vee(C M(C) \wedge$ $\langle x, y\rangle \in$ Coupling $) \vee \exists e(E M(e) \wedge$ element_of $(e, C) \wedge\langle x, y\rangle \in$ Mapping $)\}$.

The previous definition shows that the vertex set $\left(V_{C}\right)$ of $C C G$ is composed of all input and output Interfaces of the high level model, and $E_{C}$ is composed of all the Mappings edges and Coupling edges. If there is a Mapping or Coupling between two Interfaces, the two vertices are adjacent.

Definition 2 (Maps $_{C}$ and Couples ${ }_{C}$ of $C C G$ ). Consider the following:

if component $C$ is an $E M$, Maps $_{C}=$ Mapping $_{C}$, Couples $_{C}=\emptyset$;

if component $C$ is a $C M, M_{a p s_{C}}=\sum_{e \in C}$ Mapping, Couples $_{C}=$ Coupling $_{C}$.

Deduction 1. The underlying graph of $G_{C}=\left\langle V_{C}, E_{C}\right\rangle$ is a bipartite graph.

Ignoring the direction of all the edges of the directed $C C G$, we can get its underlying graph. We can prove that the underlying graph of $C C G$ is a bipartite graph.

Let $X$ = input_interface $(C), Y=$ output_interface $(C)$,

$=>V=X \cup Y$ and $X \cap Y=\emptyset$,

$=>X$ and $Y$ is 2-partition of $V_{C}$.

According to Definition 1,

$\forall x \forall y\left(x y \in E\left(G_{C}\right) \rightarrow(\exists e(E M(e) \wedge\langle x, y\rangle \in\right.$ Mapping $\left._{e}\right) \vee\left(C M(C) \wedge\langle x, y\rangle \in\right.$ Coupling $\left.\left._{C}\right)\right)$.
According to Definition 2,

$$
\begin{aligned}
= & \forall x \forall y\left(x y \in E\left(G_{C}\right) \rightarrow((x \in X \wedge y \in Y) \vee(x \in Y \wedge y \in\right. \\
& X)), \\
= & G_{C}=\left\langle V_{C}, E_{C}\right\rangle \text { is a bipartite graph. }
\end{aligned}
$$

The previous deduction means that the Mapping connects the input and output Interfaces of an EM, and Coupling connects the input and output Interfaces between EMs and/or $C M s$. The vertices of $X$ are independent of each other, and vertices of $Y$ are also independent.

Definition 3 (Information Tracking). Let $G_{C}=\left\langle V_{C}, E_{C}\right\rangle$ be the $C C G$ of $C, x_{0} \in V\left(G_{C}\right), x_{k} \in V\left(G_{C}\right)$, if

$P=x_{0} m_{1} x_{1} m_{2} x_{2} \cdots m_{k} x_{k} \wedge \forall i=1,2, \ldots, k\left(m_{i}=\right.$ $\left.\left\langle x_{i-1}, x_{i}\right\rangle\right) \wedge \forall i \forall j\left(i \neq j \rightarrow x_{i} \neq x_{j}\right)$.

Then $P$ is Information Tracking in $G_{C} . x_{0}$ and $x_{k}$ are the start and end points of $P$, referred to as startPoint st $_{P}$ and endPoint ${ }_{P}$ respectively.

Using the terminology of graph theory, Information Tracking can be described as follows:

Vertex $i$ and $j(i \neq j)$ belong to $G_{C}$, and $P$ is a primary path from $i$ to $j$ without repetitive vertices. If any adjacent vertex of $x$ is from the same EM with $x$, then it is an Information Tracking of $G_{C}$.

When there are only two vertices in the $M a p s_{C}$ or Couples $_{C}$, we can easily get the following deduction.

Deduction 2. Mapping and Logical Relation are both a kind of Information Tracking.

We can see from Definition 3 that Information Tracking is a directed path, the direction of $M a p s_{C}$ is always from the input Interface to the output Interface, while the direction of Couples $_{C}$ is from output to input. In the Information Tracking $P$, the edges of $\mathrm{Maps}_{C}$ and Couples ${ }_{C}$ appear alternately.

Deduction 3. Information Tracking $P \in$ InfoPath $_{C}$ and $P=$ $x_{0} m_{1} x_{1} m_{2} x_{2} \cdots m_{k} x_{k}, i \in\{1,2, \ldots k\}$; if $m_{1} \in$ Maps $_{C}$ then $m_{i} \in \operatorname{Maps}_{C}$ if and only if $i \equiv 1(\bmod 2)$ and $m_{i} \in$ Couples $_{C}$ if and only if $i \equiv 0(\bmod 2)$; if $m_{1} \in$ Couples $_{C}$ then $m_{i} \in$ Couples $_{C}$ if and only if $i \equiv 1(\bmod 2)$ and $m_{i} \in M a p s_{C}$ if and only if $i \equiv 0(\bmod 2)$.

Deduction $1=>$ the underlying graph of $G_{C}$ is a bipartite graph,

Deduction $2=>$ in Information Tracking $P$, the edges of $\mathrm{Maps}_{\mathrm{C}}$ and Couples $\mathrm{C}_{\mathrm{C}}$ appear alternately.

Assume that $x y$ and $y z$ are two adjacent edges of $P$, a primary path. So, $x \neq z$.

If $x y \in M a p s_{C}$, then $x$ and $y$ are the input and output of an $\operatorname{EM}(c)$. The vertices $x$ and $z$ are adjacent to $y$.

$=>$ In $x$ and $z$, one must belong to $\operatorname{EM}(c)$, and $x \neq z$.

$=>z$ must not be the Interface of $\operatorname{EM}(c)$, and it must belong to other $E M / C M$. 


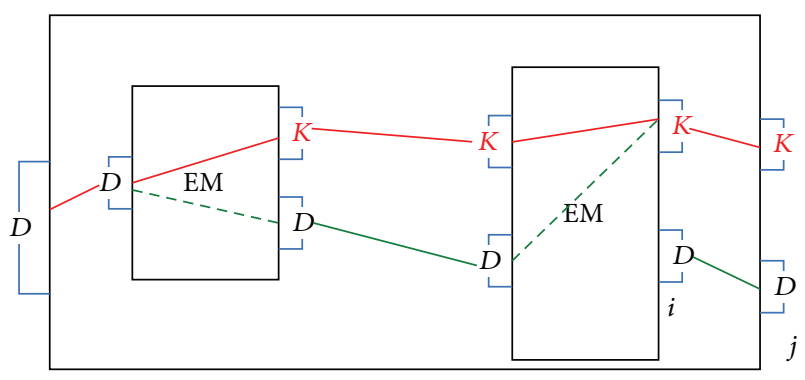

Figure 6: An Information Tracking composed by alternative Mapping and Coupling.

The underlying graph of $G_{C}$ is a bipartite graph, and in Information Tracking $P$, the edges of $M a p s_{C}$ and Couples appear alternately.

$=>z$ is a input Interface.

$=>y z \in$ Couples $_{C}$.

If $x y \in$ Couples $_{C}$, then $x$ and $y$ are the input and output of two different components. Assume that $y$ belongs to $E M(c 1)$.

$\Rightarrow$ In $x$ and $z$, there must be one belonging to $\operatorname{EM}(c 1)$, and $x \neq z$.

$=>z$ must not be the Interface of $E M(c 1)$, and it belongs to the other EM.

The underlying graph of $G_{C}$ is a bipartite graph, and, in Information Tracking $P$, the edges of $\mathrm{Maps}_{C}$ and Couples appear alternately.

$=>z$ is a output Interface.

$\Rightarrow y z$ is a Mapping of the other $E M, y z \in M a p s_{C}$.

$=>P$ is an uninterrupted path composed of alternative Mapping and Coupling. It can also be expressed by alternative input and output Interface, as shown in Figure 6.

The vertices in $P$ are independent of each other and $i \neq j$, and $\forall_{i} \forall_{j}\left(i \neq j \rightarrow x_{i} \neq x_{j}\right)$.

$=>P$ is a directed path without repetitive edges and there is no closed loop in $P$.

$\Rightarrow$ If $m_{1} \in \operatorname{Maps}_{C}, m_{i} \in \operatorname{Maps}_{C}$ if and only if $i \equiv 1(\bmod$ $2)$ and $m_{i} \in$ Couples $_{C}$ if and only if $i \equiv 0(\bmod 2)$, and if $m_{1} \in$ Couples $_{C}, m_{i} \in$ Couples $_{C}$ if and only if $i \equiv 1(\bmod 2)$ and $m_{i} \in \operatorname{Maps}_{C}$ if and only if $i \equiv 0(\bmod$ 2).

Definition 4 (Derivable Port and Underivable Port). $G_{C}=$ $\left\langle V_{C}, E_{C}\right\rangle$ is the CCG of $C, x \in$ output_interface $C_{C}$. If $\exists P\left(P \in\right.$ InfoPath $_{C} \wedge P=x_{0} m_{1} x_{1} m_{2} x_{2} \cdots m_{k} x \wedge x \in$ input interface $\left._{C}\right)$ ), then $x$ is a Derivable Port of $C$, or $x$ is an Underivable Port (referred to as DerivablePorts $(C)$ and UnderivablePorts (C), resp.).

Both Derivable Ports and Underivable Ports are output Ports. For output Ports, there are input Ports connected to it

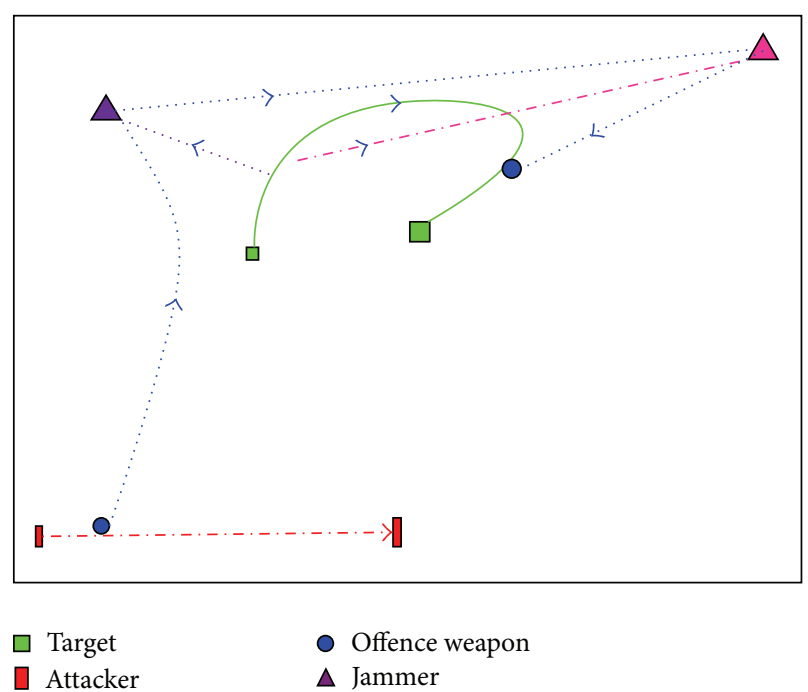

FIgURE 7: Scenario of the stochastic defense simulation system.

through an Information Tracking, but there is no such input for an Underivable Port. The internal mechanism and status of a black-box model are normally undetectable. Some outputs might be generated without any inputs and the only reason for this is due to the internal state transfer driven by time. That is why an underivable Port is needed.

Deduction 4. $G_{C}=\left\langle V_{C}, E_{C}\right\rangle$ is the CCG of $C, x \in$ DerivablePorts $(\mathrm{C}) . \exists \mathrm{e}(\mathrm{e} \in \mathrm{EM} \wedge$ element_of $(\mathrm{e}, \mathrm{C}) \wedge$ $x \in$ UnderivablePorts $(C))$ or $\exists \mathrm{e}(\mathrm{e} \in \mathrm{EM} \wedge$ element_of $(\mathrm{e}, \mathrm{C}) \wedge$ $\exists x_{0} \exists \mathrm{P}\left(\mathrm{P} \in \operatorname{InfoPath}(\mathrm{C}) \wedge x_{0} \in\right.$ UnderivablePorts $(C) \wedge x_{0}=$ startPoint $_{P} \wedge x=$ endpoint $\left._{P}\right)$ ).

In a white-box $C M(C)$, let $j$ be the Underivable Port of $G_{C}$. Every output Port of $C M(C)$ is connected with an output Port of an internal EM $(C)$ by Coupling. Assume that Port $j$ of $C M(C)$ is connected with output Port $i$ of $E M(C)$, as shown in Figure 4. We will prove that Port $i$ is an Underivable Port by reducing it to absurdity.

Assume that Port $i$ is a derivable Port, and then there is an Information Tracking in $G_{C}$. Port $i$ is the endpoint of $P$.

Port $i$ is the output Port of $\operatorname{EM}(C)$.

$=>$ The last edge of $P$ must belong to $\operatorname{Maps}_{C}$ (Deduction 3).

$=>P^{\prime}=P \cup\{i j\}$ is another Information Tracking in $G_{C}$ and at least one input Port of $P^{\prime}$ comes from $G_{C}$.

$\Rightarrow$ The end point $j$ of $P^{\prime}$ is a derivable Port. This is contradictory.

$=>$ Port $i$ is an Underivable Port.

$=>$ Underivable Port exists in an EM, and a Port connected to an Underivable Port by Coupling is also an Underivable Port.

In summary, we can see that 


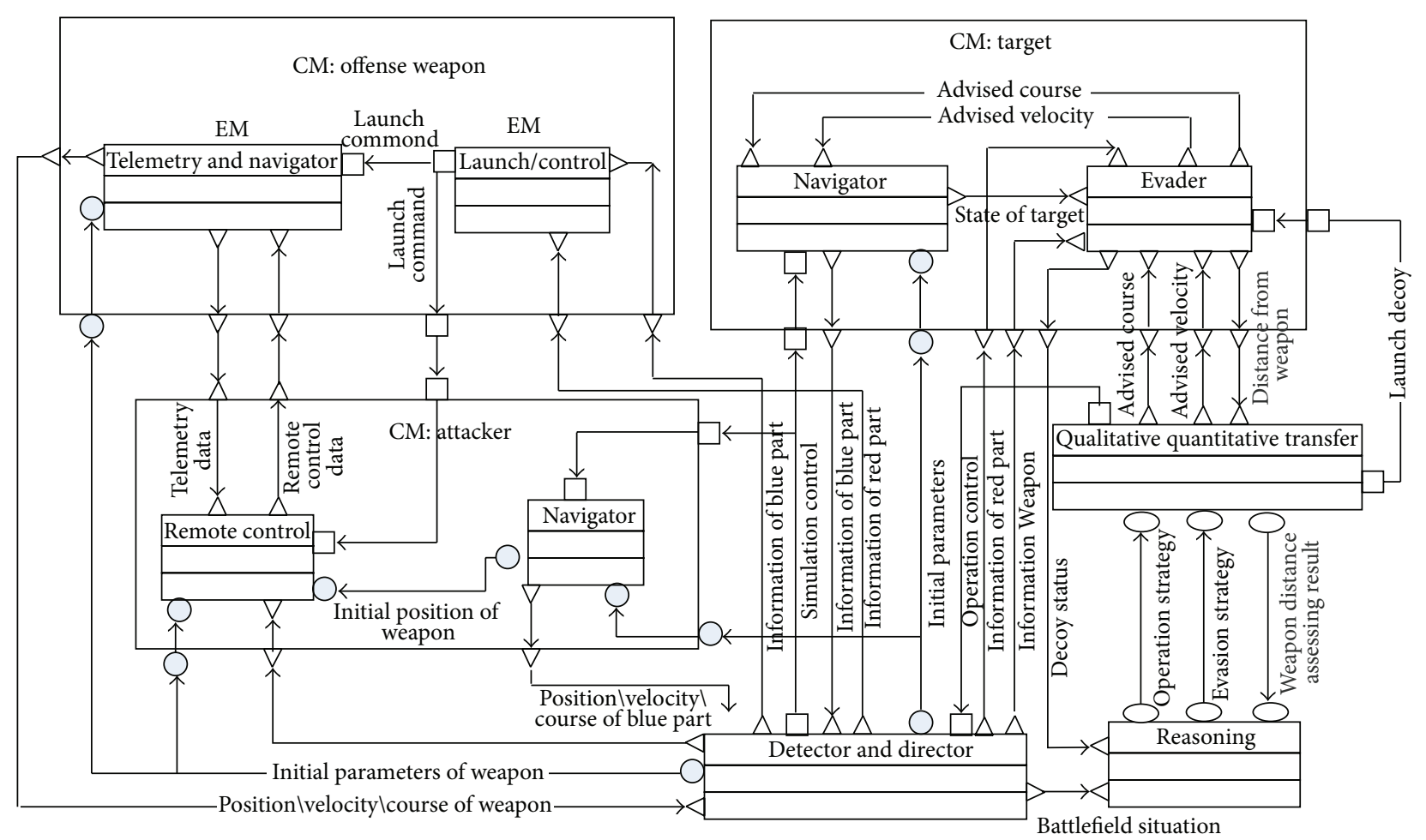

FIGURE 8: Qualitative and quantitative combined models of the stochastic defense simulation system.

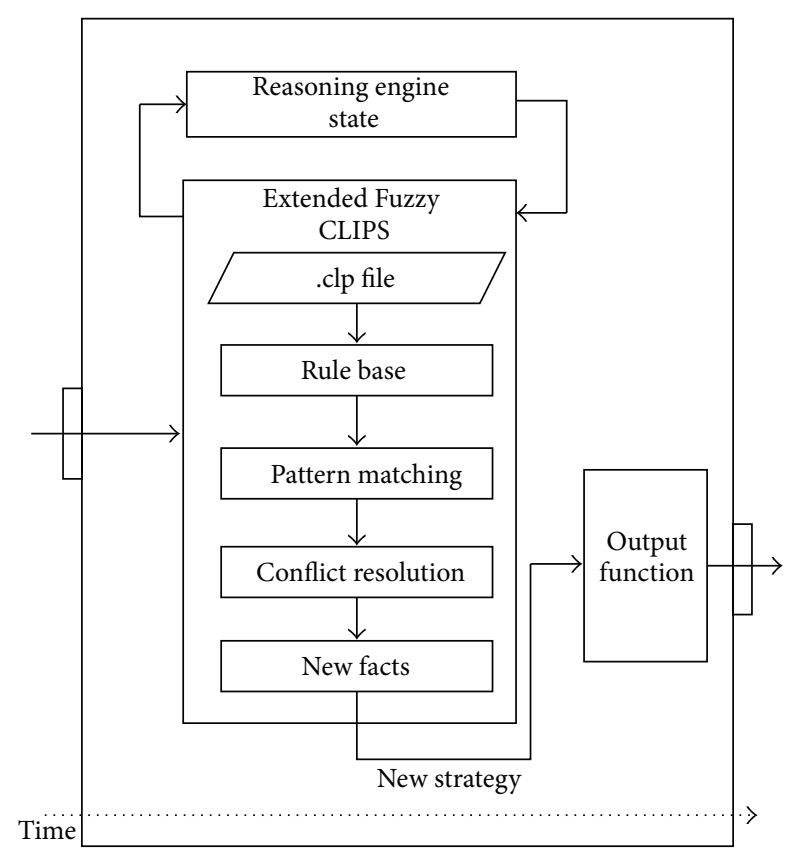

FIGURE 9: The reasoning EM, extended Fuzzy CLIPS as the reasoning engine.

$E M \sqcap$ interface $\equiv \exists$ has_a.(Init) $\sqcap \exists h a s_{-} a .\left(i P_{d}\right) \sqcap \exists h a s_{-}$ a. $\left(i P_{k}\right) \sqcap \exists$ has_a. $\left(i P_{e}\right) \sqcap \exists h a s_{\_} a .\left(o P_{d}\right) \sqcap \exists h a s \_a .\left(o P_{k}\right) \sqcap$ $\exists$ has_a. $\left(o P_{e}\right)$

$C M \sqcap$ interface $\equiv \exists$ has_a. (Init) $\sqcap \exists$ has_a. $\left(i P_{d}\right) \sqcap \exists h a s_{-}$ $a .\left(i P_{k}\right) \sqcap \exists h a s_{\_} a .\left(i P_{e}\right) \sqcap \exists h a s_{-} a .\left(o P_{d}\right) \sqcap \exists h a s_{\_} a .\left(o P_{k}\right) \sqcap$ $\exists$ has_a. $\left(o P_{e}\right)$

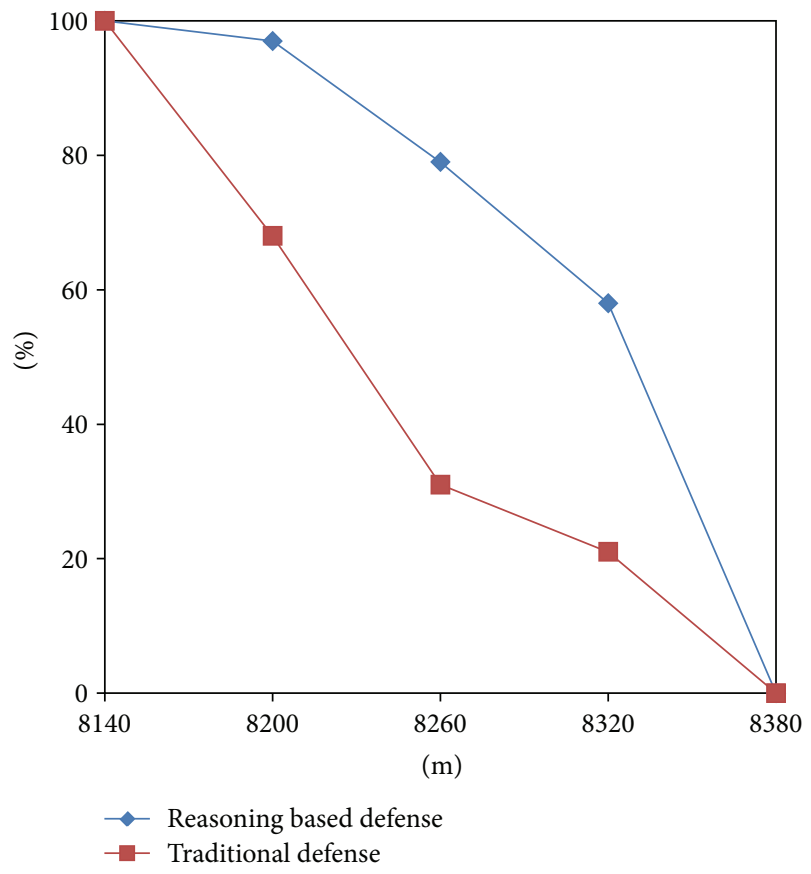

FIGURE 10: Survival probability of target.

=> EM $\sqcap$ interface $\equiv C M \sqcap$ interface.

This means that EM and CM have the same schema, and the $H L M$ is self-closed. 


\section{A Stochastic Defense Simulation System}

5.1. The Scenario and Integrated Models. In the simulation, the attacker and the target patrol in the same area, and both of them have detecting ability. As the distance between the two sides becomes shorter, the attacker will find the target and launch its offense weapon which will seek the target using its detector. The offense weapon will rush out with full speed when it detects the target. After detecting the offense weapon, the target will launch decoy or evade with different direction and speed according to the defense strategy. The scenario is as follows (Figure 7). Many previous researches carried out the same scenario; however, most of them focused on using fuzzy logic to make the decision [1] in a specific application or evading in a fixed manner [27].

The system is modeled using the proposed specification. We designed nine EMs as shown in Figure 8. There are three CMs, Attacker, Target, and Offense Weapon, which are composed by two $E M$ models, respectively. The data, event, and knowledge interactions among the CMs/EMs are also given in the figure. Different shapes are used to describe different types of Ports. The circle, square, triangle, and oval Ports represent initialize port, event port, data port, and knowledge port, respectively. What should be pointed out is that only Ports and Couplings are given in the figure, not the PortItems and Mapping. Mappings are inside the EM and are invisible from the outside. Due to the space, the dynamic behavior and schedule of $C M / E M$ will be treated as a blackbox and will be discussed in the future.

5.2. Optimization of the Defense Simulation. In our simulation, defense strategy and simulation operation strategy are decided by reasoning EM based on the real-time battlefield situation and expert experience to optimize the simulation result. We have several evasion strategies, such as launching a decoy at specific position with a reasonable direction and moving mutely with higher speed alone against_direction, depending on the battlefield situation, the decoy status, and distance between the offence weapon and target. Different simulation strategies could be adopted in different situations to optimize the operating efficiency. When the attacker is far away from the target and any other special task, the simulation can run with super-real-time speed (in speedup status); only some staple detectors in work and many functionalities will not be executed or executed in less time (in light_caculate status). The simulation time will slow down when the attacker gets closer to the target and more powerful detector will be on duty.

The defense strategy and simulation operation strategy are decided by a reasoning EM. The detail is as follows (Figure 9). We proposed a new fuzzy-reasoning algorithm based on confidence fuzzy rules and embedded it into Fuzzy CLIPS. The extended Fuzzy CLIPS is encapsulated into the $E M$ as a reasoning engine. The rules coming from expert knowledge are stored as a file $\left({ }^{*}\right.$.clp) and will be loaded to the rule base. At running time, different strategies will be made according to the battlefield situation. Some of the confidence fuzzy rules are as follows.
Rule 1. IF Weapon_distance_medium AND Decoyl_ready THEN Change_Direction_with_large_angle AND evade_ mutely AND Launch_Decoyl, Confidence: 0.85 .

Rule 2. IF Weapon_distance_s short THEN Evade_full_speed, Confidence: 0.9 .

Rule 3. IF Distance_between_attacker_target_far THEN simulation_speedup AND light_caculate, Confidence: 0.9.

5.3. Simulation Results and Analysis. The initial speeds of attacker and target are both $18 \mathrm{~m} / \mathrm{s}$. When the offence weapon is launched, its initial speed is $20 \mathrm{~m} / \mathrm{s}$. The detection range is $1.5 \mathrm{~km}$ apart. The initial distance between attacker and target is $8 \mathrm{Km}$. The simulation is executed in two situations. First, defense strategy is fixed as evade_full_speed or Lauch_Decoy1 or Lauch_Decoy2 randomly and running speed is also fixed. Secondly, the reasoning model will be used. The simulation time and data communication can be saved significantly at the beginning because of simulation_speedup and light_caculate strategy.

In fact, the voyage of the weapon is one of the key factors in the survival probability of the target. If the voyage is long enough, the target will be destroyed with probability 1 . If it is short, the weapon will exhaust before catching the target. We set different voyages for the weapon, and the simulation is executed 20 times for each voyage in the two situations. The average survival probability is shown in Figure 10. We can see that when the voyage is shorter than $8140 \mathrm{~m}$, the target will always survive, and if the voyage is longer than $8380 \mathrm{~m}$, the target will be destroyed absolutely. Between $8380 \mathrm{~m}$ and $8140 \mathrm{~m}$, the probability of survival is higher, when we simulate based on qualitative and quantitative integrated models.

\section{Conclusions and Future Works}

In this paper, we have proposed a new specification to modeling qualitative and quantitative hybrid system for stochastic simulation and optimization. The new specification is defined at three levels and its self-closed feature is proven to be self-closed formally. The definition of factors needed to describe the integrated models and corresponding Mapping and Coupling is presented in detail. This provides a new way to take advantage of qualitative models in stochastic simulation. A stochastic simulation defense system was modeled and realized using the proposed specification; a reasoning engine is encapsulated as a qualitative $E M$ and interacts with quantitative models at running time. The result shows that the hybrid models can optimize the stochastic simulation significantly on both the execution process and the performance.

As future works, the dynamic behavior and schedule engine of qualitative and quantitative integrated models for stochastic simulation in different application should be a great work that will be promoted in detail and verified. Also, more working on the integration relationship, interaction, 
and time management of qualitative and quantitative stochastic models are significant for the new specification.

\section{Acknowledgments}

This work was supported by Major Basis Research under Grant no. C0420110005 in China. The authors acknowledge and appreciate all the team members. They are also grateful to editors and reviewers for their constructive comments, which helped improve this paper greatly.

\section{References}

[1] M.-J. Son and T.-W. Kim, "Torpedo evasion simulation of underwater vehicle using fuzzy-logic-based tactical decision making in script tactics manager," Expert Systems with Applications, vol. 39, no. 9, pp. 7995-8012, 2012.

[2] L. I. Wenwei, "Research on the development and application of the method of qualitative simulation," System Simulation Technology, vol. 4, no. 2, pp. 71-74, 2008 (Chinese).

[3] S. Fan, B.-H. Li, X.-D. Chai, B.-C. Hou, and T. Li, "Studies on complex system qualitative and quantitative synthetically modeling technologies," Journal of System Simulation, vol. 23, no. 10, pp. 2227-2233, 2011.

[4] S. Fan, B.-H. Li, X.-D. Chai, and X.-D. Huang, "Studies on qualitative and quantitative integration model computing technology," Journal of System Simulation, vol. 23, no. 9, pp. 1980-1984, 2011.

[5] F. E. Cellier, "Mixed quantitative and qualitative simulation in modelica," in Proceedings of the 7th Modelica Conference, pp. 8695, Como, Italy, September 2009.

[6] M. Samejima, M. Akiyoshi, K. Mitsukuni, and N. Komoda, "Business scenario evaluation using monte carlo simulation on qualitative and quantitative hybrid model," Electrical Engineering in Japan, vol. 170, no. 3, pp. 9-18, 2010.

[7] T. Li, B.-H. Li, X.-D. Chai, and S. Fan, "Research on knowledge modeling and joint simulation method of complex qualitative system," Journal of System Simulation, vol. 23, no. 6, pp. 12561260, 2011.

[8] V. Varho and P. Tapio, "Combining the qualitative and quantitative with the $\mathrm{Q}_{2}$ scenario technique-the case of transport and climate," Technological Forecasting and Social Change, vol. 80, no. 4, pp. 611-630, 2013.

[9] H.-g. Liu, J.-j. Wu, and Q.-z. Chen, "Fault diagnosis reasoning based on integration of qualitative reasoning and quantitative simulation," Journal of System Simulation, vol. 15, no. 5, pp. 689692, 2003.

[10] A.-F. Shi, Q.-Z. Wu, K. Huang, and H. Zheng, "Study on characteristic of qualitative models and simulation about complex weapon system," Journal of System Simulation, vol. 18, no. 2, pp. 591-593, 2006 (Chinese).

[11] K. Ogata, Modern Control Engineering, Prentice Hall, Upper Saddle River, NJ, USA, 5th edition, 2009.

[12] K. Edström, "Simulation of Newton's pendulum using switched bond graphs," 1998, http://citeseerx.ist.psu.edu/.

[13] R. David and H. Alla, "On hybrid Petri nets," Discrete Event Dynamic Systems, vol. 11, no. 1-2, pp. 9-40, 2001.

[14] D. Karnopp, R. Rosenberg, Perelson, and S. Alan, System Dynamics: A Unified Approach, John Wiley \& Sons, New York, NY, USA, 1976.
[15] B. P. Zeigler, H. Praehofer, and T. G. Kim, Theory of Modeling and Simulation, Academic Press, New York, NY, USA, 2nd edition, 2000.

[16] B. H. Li, X. Chai, X. Yan, and B. Hou, Multi-Disciplinary Virtual Prototype Modeling and Simulation Theory and Application, Nova Science, New York, NY, USA, 2012.

[17] L. Ironi and L. Panzeri, "Qualitative simulation of nonlinear dynamical models of gene-regulatory networks.," in Proceedings of the 22nd International Workshop on Qualitative Reasoning, University of Colorado, Boulder, Colo, USA, 2008.

[18] C.-X. Shao and F.-Z. Bai, "Technology of qualitative simulation and its application," Journal of System Simulation, vol. 16, no. 2, pp. 202-208, 2004 (Chinese).

[19] Y. Hashiura, T. Fujimoto, and T. Matsuo, "Qualitative simulation models for non-autonomous systems," in Proceedings of the 21st International Conference on Systems Engineering (ICSEng '11), pp. 392-397, August 2011.

[20] H. Yang, B. Hu, and L. Zhang, "Analysis for the quality risk evolution of highway engineering construction based on qualitative simulation," in Proceedings of the International Conference on Computer Science and Service System (CSSS '11), pp. 212-215, June 2011.

[21] H. Wang and W.-J. Li, "Study on the Qualitative simulationbased customer churn prediction," in Proceedings of the International Symposium on Information Engineering and Electronic Commerce (IEEC '09), pp. 528-532, May 2009.

[22] B. Hu and R. Xiao, "Qualitative simulation for complex systems," System Simulation Technology, vol. 2, no. 1, pp. 1-10, 2006 (Chinese).

[23] R.-w. Dai, "The proposal and recent development of metasynthetic method from qualitative to quantitative," Chinese Journal of Nature, vol. 31, no. 6, pp. 311-314, 2009 (Chinese).

[24] F. Baader, D. L. McGuinness, D. Nardi, and P. Patel-Schneider, The Description Logic Handbook: Theory, Implementation and Applications, Cambridge University Press, Cambridge, Mass, USA, 2003.

[25] F. Baader, C. Lutz, M. Miličić, U. Sattler, and F. Wolter, "Integrating description logics and action formalisms: First results," in Proceedings of the 20th National Conference on Artificial Intelligence and the 17th Innovative Applications of Artificial Intelligence Conference (AAAI '05/IAAI '05), pp. 572-577, July 2005.

[26] A. Borgida, "On the relative expressiveness of description logics and predicate logics," Artificial Intelligence, vol. 82, no. 1-2, pp. 353-367, 1996.

[27] D.-Y. Cho, M.-J. Son, J.-H. Kang et al., "Analysis of a submarine’s evasive capability against an antisubmarine warfare torpedo using devs modeling and simulation," in Proceedings of the Spring Simulation Multiconference and DEVS Integrative Modeling and Simulation Symposium (DEVS), pp. 307-315, Norfolk Marriott Waterside, Norfolk, Virginia, USA, 2007. 


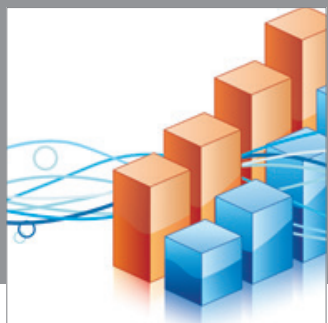

Advances in

Operations Research

mansans

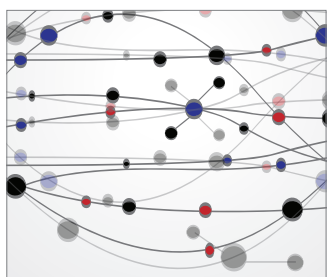

The Scientific World Journal
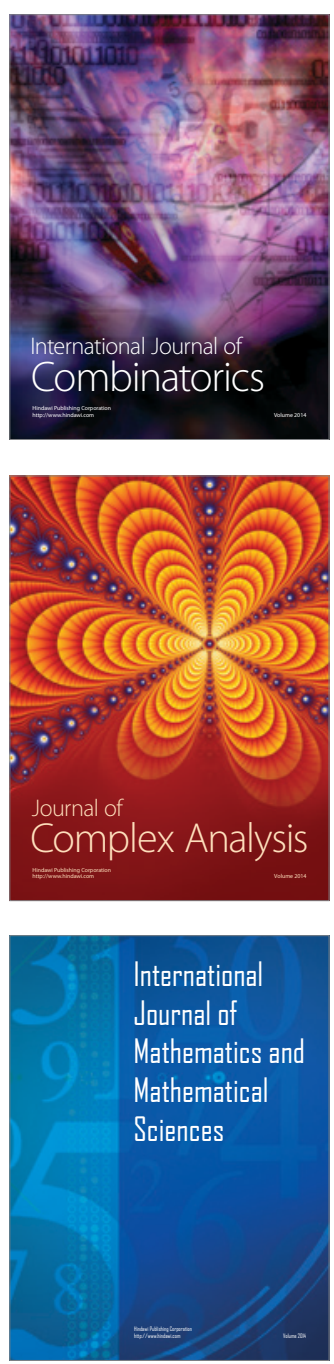
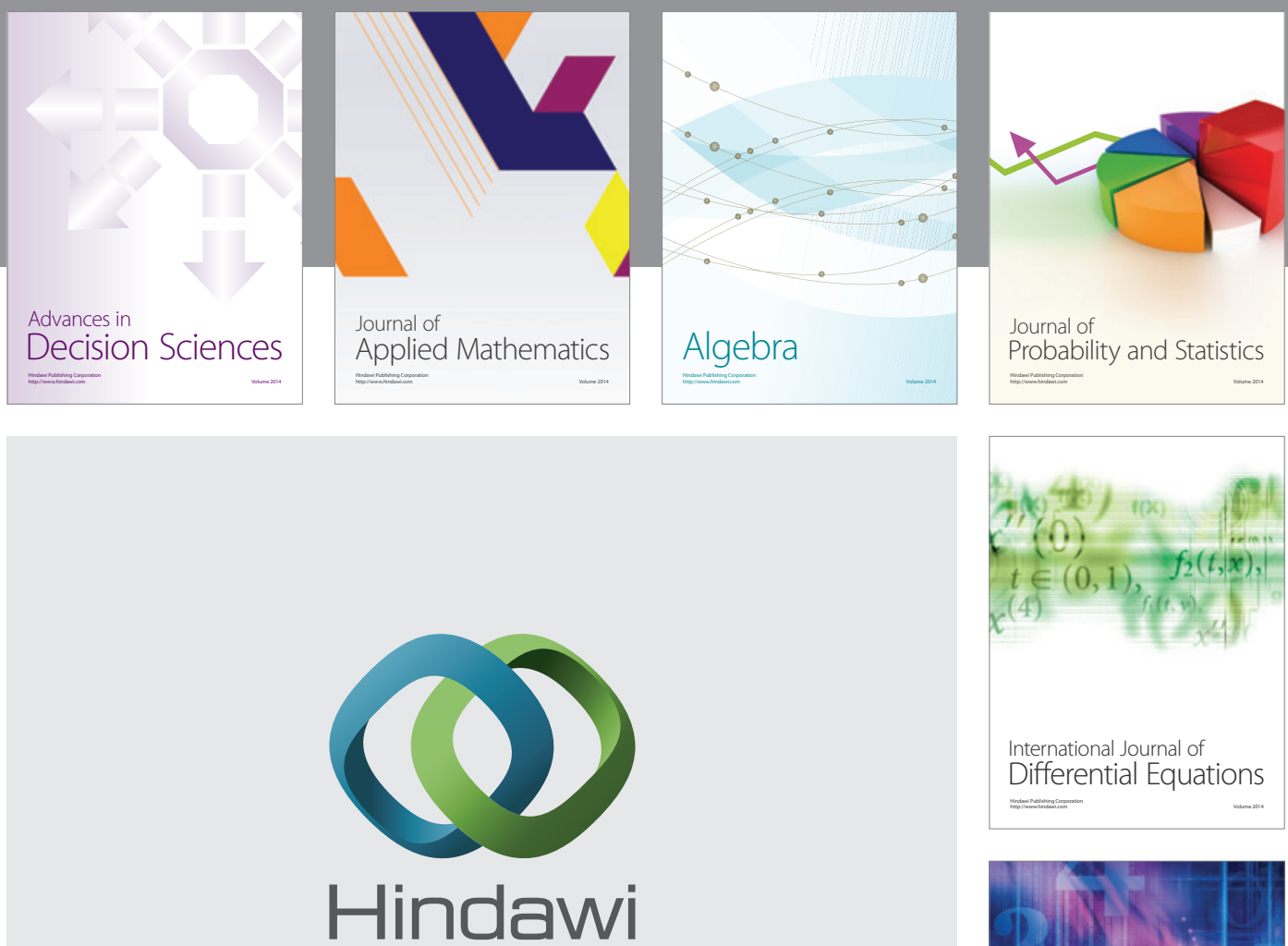

Submit your manuscripts at http://www.hindawi.com
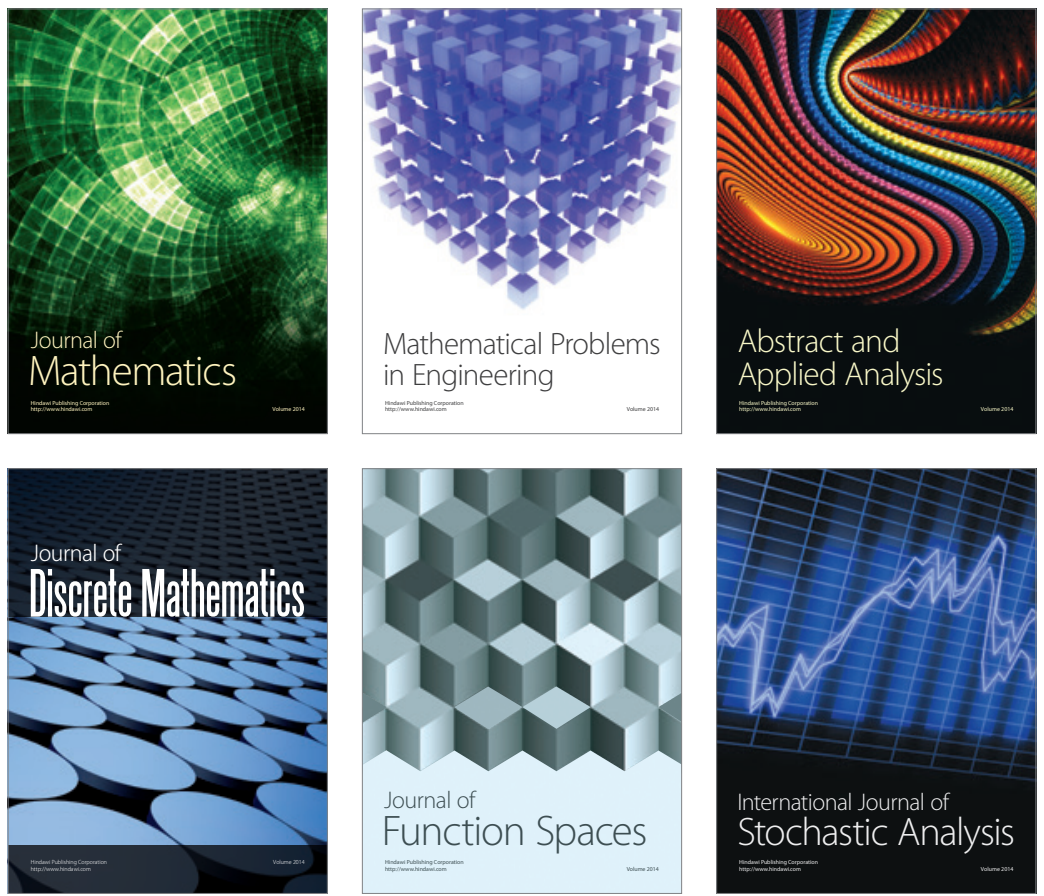

Journal of

Function Spaces

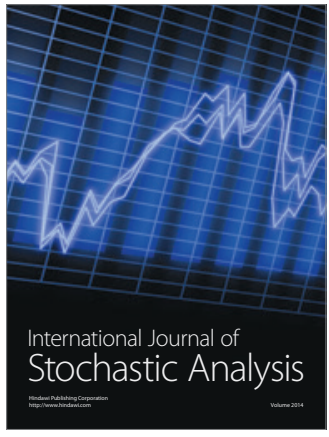

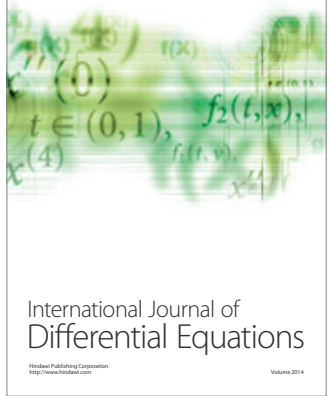
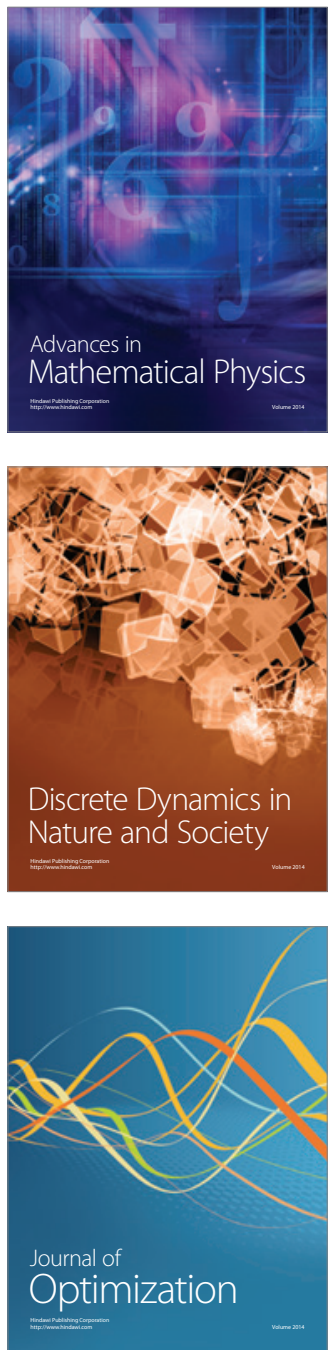